\title{
Logarithmic norms and regular perturbations of differential equations
}

\author{
Dedicated to Yuri Kozitsky on the occasion of his 70th birthday
}

\begin{abstract}
In this note we explore the concept of the logarithmic norm of a matrix and illustrate its applicability by using it to find conditions under which the convergence of solutions of regularly perturbed systems of ordinary differential equations is uniform globally in time.
\end{abstract}

\section{Introduction}

The concept of the logarithmic norm of a matrix, also known as a logarithmic derivative, or the logarithmic Lipschitz constant for nonlinear operators, was introduced independently by Dahlquist [3] and Lozinski [6], whose aim was to estimate the error of discretization in numerical analysis of differential equations, see e.g. [5, Section I.10]. This "norm" (which is not really a norm in the sense of functional analysis as it can take negative values) is a measure of the dissipativity of an operator. It has, nevertheless, many norm-like properties that allow to use the dissipative terms in the original problem to generate negative terms in the error estimates. In this way the error estimates are reduced to linear differential inequalities that reflect

2010 Mathematics Subject Classification. 34E10, 47A30.

Key words and phrases. Logarithmic norm, stability, regular perturbations of differential equations.

Research partially supported by National Science Centre, Poland, grant 2017/25/B/ST1/00051. 
the structure of the original problem in a much better way than the Gronwall inequality. In particular, in many cases it yields estimates uniform in time, remedying thus one of the main drawbacks of the Gronwall inequality, namely that the constants grow exponentially in time.

It turns out that this feature of the logarithmic norm can be used to provide uniform globally in time estimates for solutions to perturbed systems of differential equations. To explain the problem, let us recall a classical regular perturbation result.

Theorem 1.1 ([17, Theorem VI, Chapter III §12]). Let $J$ be a compact interval with $t_{0} \in J$ and let the function $\boldsymbol{x}=\boldsymbol{x}_{0}(t)$ be a solution of the initial value problem

$$
\boldsymbol{x}^{\prime}=\boldsymbol{f}(t, \boldsymbol{x}) \text { in } J, \quad \boldsymbol{x}\left(t_{0}\right)=\stackrel{\circ}{\boldsymbol{x}} .
$$

The $\alpha$-neighbourhood $(\alpha>0)$ of graph of $\boldsymbol{x}_{0}$ (definition: the set of all points $(t, \boldsymbol{x})$ with $\left.t \in J,\left|\boldsymbol{x}-\boldsymbol{x}_{0}(t)\right| \leq \alpha\right)$ will be denoted by $S_{\alpha}$. Suppose there exists $\alpha>0$ such that $\boldsymbol{f}(t, \boldsymbol{x})$ is continuous and Lipschitz in $\boldsymbol{x}$ uniformly in $t$ in $S_{\alpha}$. Then the solution $\boldsymbol{x}_{0}(t)$ depends continuously on the initial values and on the right-hand side $\boldsymbol{f}$. In other words: For every $\epsilon>0$, there is $\delta>0$ such that if $\boldsymbol{g}$ is continuous in $S_{\alpha}$ and the inequalities

$$
|\boldsymbol{g}(t, \boldsymbol{x})-\boldsymbol{f}(t, \boldsymbol{x})|<\delta \text { in } S_{\alpha}, \quad|\dot{z}-\stackrel{\boldsymbol{x}}{ }|<\delta
$$

are satisfied, then every solution $\boldsymbol{z}(t)$ of the perturbed initial value problem

$$
\boldsymbol{z}^{\prime}=\boldsymbol{g}(t, \boldsymbol{z}), \quad \boldsymbol{z}\left(t_{0}\right)=\stackrel{\boldsymbol{z}}{ } .
$$

exists in all of $J$ and satisfies the inequality

$$
\left|\boldsymbol{z}(t)-\boldsymbol{x}_{0}(t)\right|<\epsilon \text { in } J .
$$

This result does not give, however, a satisfactory answer as far as the long term dynamics is concerned. In fact, even if solutions to (1) converge as $t \rightarrow \infty$ to, say, a stationary point, the solutions to (3) can diverge as $t \rightarrow \infty$, or even blow-up at finite time for any $\delta>0$.

Example 1.2. Consider the family of Cauchy problems

$$
x_{\epsilon}^{\prime}=\epsilon x_{\epsilon}^{2}, \quad x_{\epsilon}(0)=1 .
$$

Then $x_{0}(t)=1, t \geq 0$, and

$$
x_{\epsilon}(t)=\frac{1}{1-\epsilon t}, \quad 0 \leq t<\frac{1}{\epsilon} .
$$

It is clear that for $\epsilon>0, x_{\epsilon}(t)$ blows up as $t \rightarrow \frac{1}{\epsilon}^{-}$. On the other hand, for any compact interval $[0, T]$ the solution $x_{\epsilon}(t)$ is defined on $[0, T]$ for $\epsilon<\epsilon_{0}=\frac{1}{T}$ and satisfies

$$
\lim _{\epsilon \rightarrow 0^{+}} x_{\epsilon}(t)=x_{0}(t)
$$


uniformly on $[0, T]$.

On the other hand, if we consider

$$
x_{\epsilon}^{\prime}=-x_{\epsilon}+\epsilon x_{\epsilon}^{2}, \quad x_{\epsilon}(0)=1,
$$

then $x_{0}(t)=e^{-t}$ and

$$
x_{\epsilon}(t)=\frac{1}{e^{t}(1-\epsilon)+\epsilon}, \quad t \geq 0 .
$$

Since

$$
\left|\frac{1}{e^{t}(1-\epsilon)+\epsilon}-e^{-t}\right|=\frac{\epsilon\left(e^{t}-1\right)}{e^{t}\left(e^{t}(1-\epsilon)+\epsilon\right)} \leq \epsilon \frac{e^{-t}}{1-\epsilon},
$$

we see that

$$
\lim _{\epsilon \rightarrow 0^{+}} x_{\epsilon}(t)=x_{0}(t)
$$

uniformly on $[0, \infty)$.

We observe that, contrary to the first case, in the second case $x^{*}=0$ is the hyperbolic equilibrium of the limit problem. To simplify the discussion, we slightly modify (1) to

$$
\boldsymbol{x}_{\epsilon}^{\prime}=\boldsymbol{f}\left(\boldsymbol{x}_{\epsilon}, \epsilon\right) \text { in } J, \quad \boldsymbol{x}_{\epsilon}\left(t_{0}\right)=\stackrel{\circ}{\boldsymbol{x}}_{\epsilon},
$$

assume that $\boldsymbol{f}$ is differentiable with respect to all variables and $\boldsymbol{f}\left(\boldsymbol{x}^{*}, 0\right)=0$. Then, if the Jacobian $\mathcal{J} \boldsymbol{f}\left(\boldsymbol{x}^{*}, 0\right)$ is not singular, then the Implicit Function Theorem implies that for some $\epsilon_{0}>0$ there is a differentiable family $\boldsymbol{x}^{*}(\epsilon), \epsilon \in\left(-\epsilon_{0}, \epsilon_{0}\right)$, of solutions to $\boldsymbol{f}\left(\boldsymbol{x}^{*}(\epsilon), \epsilon\right)=0$. If, in addition, $\boldsymbol{x}^{*}$ is a hyperbolic equilibrium, then the theorem on continuous dependence of the roots of a polynomial on its coefficients, see e.g. [8, 15], shows that for sufficiently small $\epsilon$ the equilibria $\boldsymbol{x}^{*}(\epsilon)$ are of the same type as $\boldsymbol{x}^{*}$. In other words, for regularly perturbed problems (7) we have the convergence of its solutions on any finite time interval to the solution of the limit problem (with $\epsilon=0$ ). Moreover, provided the equilibria of the limit problem are hyperbolic, (7) has the same structure and the type of the equilibria, and these equilibria converge to the equilibria of the limit problem. However, this does not prove that the interval of convergence of the solutions can be extended to $\left[t_{0}, \infty\right)$. To prove this fact, some uniform estimates of $\boldsymbol{x}_{\epsilon}$ for large times would be required. The aim of this note is to show how the logarithmic norm can be used to prove such estimates.

Acknowledgements. The author is grateful to Dr. S. Shindin for drawing his attention to the concept of the logarithmic norm. 


\section{Basic mathematical tools}

As the main idea of using the logarithmic norm to error estimates consists in deriving an appropriate differential inequality for the norm of the error, we must specify in what sense that norm can be differentiated. In this section we discuss this issue as well as the solvability of relevant differential inequalities, mostly following [2, Chapter I].

Let $X=\mathbb{R}^{n}$ and let $\|\cdot\|$ be a norm on $X$.

Definition 2.1. Let $f:(a, b) \rightarrow \mathbb{R}$ be any function. The upper and lower right Dini derivatives of $f$ at $t \in(a, b)$ are defined, respectively, as

(8) $D^{+} f(t)=\limsup _{h \rightarrow 0^{+}} \frac{f(t+h)-f(t)}{h}, \quad D_{+} f(t)=\liminf _{h \rightarrow 0^{+}} \frac{f(t+h)-f(t)}{h}$.

Similarly, the upper and lower left Dini derivatives of $f$ are defined, respectively, as

(9) $D^{-} f(t)=\limsup _{h \rightarrow 0^{+}} \frac{f(t)-f(t-h)}{h}, \quad D_{-} f(t)=\liminf _{h \rightarrow 0^{+}} \frac{f(t)-f(t-h)}{h}$.

If $D_{+} f(t)=D^{+} f(t)$ (resp. $\left.D_{-} f(t)=D^{-} f(t)\right)$ at some $t$, then we say that $f$ has right (resp. left) hand side derivative at $t$ and denote it by $f_{+}^{\prime}(t)$ (resp. $f_{-}^{\prime}(t)$ ), or by $\frac{d}{d t^{+}} f(t)$ (resp. $\frac{d}{d t^{-}} f(t)$ ).

Due to the properties of the upper and lower limits we have

$$
D_{ \pm}^{ \pm}(f+g)=D_{ \pm}^{ \pm} f+g^{\prime}
$$

as long as $g$ is differentiable. Clearly, the formula remains correct if $g$ only has one side derivatives and $D$ is the corresponding one sided Dini's derivative. We shall be concerned with the system of differential equations

$$
\begin{aligned}
\boldsymbol{x}^{\prime}(t) & =\boldsymbol{f}(t, \boldsymbol{x}), \quad t \in(a, b) \\
\boldsymbol{x}\left(t_{0}\right) & =\boldsymbol{x}_{0},
\end{aligned}
$$

where $t_{0} \in(a, b)$.

Definition 2.2. A vector function $\boldsymbol{f}=\left(f_{1}, \ldots, f_{n}\right): D \rightarrow \mathbb{R}^{n}, D \subset \mathbb{R}^{n}$, is said to be of type $K$ (or to be quasimonotone increasing) if for each $i=1, \ldots, n$ we have $f_{i}(\boldsymbol{x}) \leq f_{i}(\boldsymbol{y})$ for any $\boldsymbol{x}, \boldsymbol{y} \in D$ with $x_{j} \leq y_{j}$ for $j \neq i$ and $x_{i}=y_{i}$.

Theorem 2.3 ([2, Theorem I.10] or [12, Theorem B.1]). Let $(t, \boldsymbol{x}) \mapsto \boldsymbol{f}(t, \boldsymbol{x})$ be continuous for $(t, \boldsymbol{x})$ in an open set $(a, b) \times \Omega$ and of type $K$ for each $t \in(a, b)$. Let $t \mapsto \boldsymbol{x}(t)$ be a solution to (11) defined on an interval $[a, b]$. If $\boldsymbol{z}$ is continuous on $[a, b]$, satisfies $D_{-} \boldsymbol{z}(t) \leq \boldsymbol{f}(t, \boldsymbol{z})$ for $a<t<b$ and $\boldsymbol{z}(a) \leq \boldsymbol{x}(a)$, then $\boldsymbol{z}(t) \leq \boldsymbol{x}(t)$ on $[a, b]$. Similarly, if $\boldsymbol{y}$ is continuous on $[a, b]$, satisfies $D^{-} \boldsymbol{y}(t) \geq \boldsymbol{f}(t, \boldsymbol{y})$ for $a<t<b$ and $\boldsymbol{y}(a) \geq \boldsymbol{x}(a)$, then $\boldsymbol{y}(t) \geq \boldsymbol{x}(t)$ on $[a, b]$. 
Corollary 2.4 ([2, Corollary to Theorem I.10]). A continuous vector function $\boldsymbol{z}$ is non-increasing on an interval $[a, b]$ if and only if $D_{-} \boldsymbol{z} \leq 0$ (or, equivalently, $D_{+} \boldsymbol{z} \leq 0$ ).

Remark 2.5. Clearly, if $D^{-} \boldsymbol{z} \leq 0$ (resp. $D^{+} \boldsymbol{z} \leq 0$ ), then the same is true for $D_{-} \boldsymbol{z}$ (resp. $D_{+} \boldsymbol{z}$ ) so also in these two cases we obtain the monotonicity of $\boldsymbol{z}$, see also [17, Appendix B.I].

Remark 2.6. Following Remark 2.5, in the statement of Theorem 2.3 the inequality $D_{-} \boldsymbol{z} \leq \boldsymbol{f}(t, \boldsymbol{z})$ can be replaced by $D_{+} \boldsymbol{z} \leq f(t, \boldsymbol{z})$. Indeed, consider

$$
\boldsymbol{u}(t)=\boldsymbol{z}(t)-\int_{a}^{t} \boldsymbol{f}(s, \boldsymbol{z}(s)) d s .
$$

Then, by (10),

$$
D_{-} \boldsymbol{u}(t)=D_{-} \boldsymbol{z}(t)-f(t, \boldsymbol{z}(t)), \quad D_{+} \boldsymbol{u}(t)=D_{+} \boldsymbol{z}(t)-f(t, \boldsymbol{z}(t)) .
$$

Since, by Corollary 2.4, $D_{+} \boldsymbol{u}(t) \leq 0$ is equivalent to $D_{-} \boldsymbol{u}(t) \leq 0$, we see that $D_{+} \boldsymbol{z} \leq f(t, \boldsymbol{z})$ implies $D_{-} \boldsymbol{z} \leq f(t, \boldsymbol{z})$ and the statement of the first part of Theorem 2.3 follows.

Similarly, in the second part of Theorem 2.3, the inequality $D^{-} \boldsymbol{y} \geq \boldsymbol{f}(t, \boldsymbol{y})$ can be replaced by $D^{+} \boldsymbol{y} \geq f(t, \boldsymbol{y})$. Indeed, consider

$$
\boldsymbol{u}(t)=\int_{a}^{t} \boldsymbol{f}(s, \boldsymbol{y}(s)) d s-\boldsymbol{y}(t) .
$$

Then, again by (10),

$$
D_{-} \boldsymbol{u}(t)=f(t, \boldsymbol{y}(t))-D^{-} \boldsymbol{y}(t), \quad D_{+} \boldsymbol{u}(t)=f(t, \boldsymbol{y}(t))-D^{+} \boldsymbol{y}(t)
$$

and Corollary 2.4 implies that $D_{+} \boldsymbol{u}(t) \leq 0$ is equivalent to $D_{-} \boldsymbol{u}(t) \leq 0$. Thus we see that $D^{+} \boldsymbol{y}(t) \geq f(t, \boldsymbol{y})$ implies $D_{-} \boldsymbol{y}(t) \geq f(t, \boldsymbol{y})$ and the statement of the second part of Theorem 2.3 follows.

The following theorem is a consequence of the convexity of the norm.

Theorem 2.7 ([17, Theorem B.IV]). If $t \mapsto \boldsymbol{u}(t)$ is continuous and has left/right derivative at $t$, then $t \mapsto\|\boldsymbol{u}(t)\|$ has left/right derivative at $t$ and

$$
-\left\|\boldsymbol{u}_{ \pm}^{\prime}(t)\right\| \leq\|\boldsymbol{u}(t)\|_{ \pm}^{\prime} \leq\left\|\boldsymbol{u}_{ \pm}^{\prime}(t)\right\| .
$$

In particular, if $\boldsymbol{u}$ is differentiable at $t=t_{0}$, then

$$
-\left\|\boldsymbol{u}^{\prime}(t)\right\| \leq\|\boldsymbol{u}(t)\|_{-}^{\prime} \leq\|\boldsymbol{u}(t)\|_{+}^{\prime} \leq\left\|\boldsymbol{u}^{\prime}(t)\right\|
$$




\section{Logarithmic, or Lozinski's, norm}

Let $A$ be an $n \times n$ matrix. Usually we shall use real matrices but most considerations are valid also in the complex case. Let $\|\cdot\|$ be a norm on $\mathbb{R}^{n}$ $\left(\right.$ or $\mathbb{C}^{n}$ ). For a given norm $\|\cdot\|$, we define the logarithmic norm of $A$ by

$$
\mu(A)=\lim _{h \rightarrow 0^{+}} \frac{\|I+h A\|-1}{h}=\left.\frac{d}{d t^{+}}\|I+t A\|\right|_{t=0} .
$$

The existence of $\mu(A)$ is ensured by Theorem 2.7. As mentioned in Introduction, $\mu(A)$ has most properties of the norm. These properties, as well as other ones relevant to this paper, are listed for instance in $[2$, Section 1 of Chapter 2], or in $[6,13,14]$. Some of them are proved in [6], while the proofs of others are scattered among sources that are not easily accessible and thus we decided to provide them here.

Lemma 3.1 ([6, Lemma 2]). For any square matrices $A$ and $B$ of the same dimension

a) For any $\alpha \geq 0$,

$$
\mu(\alpha A)=\alpha \mu(A)
$$

b)

$$
|\mu(A)| \leq\|A\|
$$

c)

$$
\mu(A+B) \leq \mu(A)+\mu(B) .
$$

Proof. a) We can write

$$
\begin{aligned}
\mu(\alpha A) & =\lim _{h \rightarrow 0^{+}} \frac{\|I+h(\alpha A)\|-1}{h} \\
& =\alpha \lim _{h \rightarrow 0^{+}} \frac{\|I+(\alpha h) A\|-1}{\alpha h}=\alpha \mu(A) .
\end{aligned}
$$

b) Similarly, by the triangle inequality for $\|\cdot\|$,

$$
\begin{aligned}
& \mu(A)=\lim _{h \rightarrow 0^{+}} \frac{\|I+h A\|-1}{h} \leq\|A\|, \\
& \mu(A)=\lim _{h \rightarrow 0^{+}} \frac{\|I+h A\|-1}{h} \geq \lim _{h \rightarrow 0^{+}} \frac{|\|I\|-h\|A\||-1}{h}=-\|A\| .
\end{aligned}
$$

c) We have

$$
\begin{aligned}
\frac{\|I+h(A+B)\|-1}{h} & =\frac{\left.\| \frac{1}{2} I+h A+\frac{1}{2} I+h B\right) \|-\frac{1}{2}-\frac{1}{2}}{h} \\
& \leq \frac{\left\|\frac{1}{2} I+h A\right\|-\frac{1}{2}}{h}+\frac{\left\|\frac{1}{2} I+h B\right\|-\frac{1}{2}}{h} \\
& =\frac{\|I+(2 h) A\|-1}{2 h}+\frac{\|I+(2 h) B\|-1}{2 h}
\end{aligned}
$$


and the result follows by passing to the limit as $h \rightarrow 0^{+}$.

Theorem 3.2.

$$
\mu(A) \leq 0 \quad \text { if and only if } \quad\left\|e^{t A}\right\| \leq 1 \text { for all } t \geq 0 .
$$

Proof. Let us consider $t \mapsto\left\|e^{t A}\right\|$. Then

$$
\begin{aligned}
\frac{\left\|e^{(t+h) A}\right\|-\left\|e^{t A}\right\|}{h} & =\frac{\left\|e^{h A} e^{t A}\right\|-\left\|e^{t A}\right\|}{h} \\
& =\frac{\left\|\left(I+h A+O\left(h^{2}\right)\right) e^{t A}\right\|-\left\|e^{t A}\right\|}{h} \\
& \leq \frac{\|I+h A\|-1}{h}\left\|e^{t A}\right\|+O(h)
\end{aligned}
$$

and hence, as $h \rightarrow 0^{+}$,

$$
\frac{d}{d t^{+}}\left\|e^{t A}\right\| \leq 0
$$

provided $\mu(A) \leq 0$. But this, by Corollary 2.4 , implies

$$
\left\|e^{t A}\right\| \leq\left\|e^{0 A}\right\|=1 .
$$

To prove the converse, we have

$$
\frac{\|I+h A\|-1}{h}=\frac{\left\|e^{h A}+O\left(h^{2}\right)\right\|-1}{h} \leq \frac{\left\|e^{h A}\right\|-1}{h}+O(h)
$$

and, letting $h \rightarrow 0^{+}$,

$$
\mu(A) \leq \frac{d}{d t^{+}}\left\|e^{t A}\right\|_{t=0} .
$$

Since $\left\|e^{t A}\right\| \leq 1=\left\|e^{0 A}\right\|$, from the definition of the right hand derivative and its existence, we infer $\left.\frac{d}{d t^{+}}\left\|e^{t A}\right\|\right|_{t=0} \leq 0$, which proves the thesis.

Corollary 3.3. We have

$$
\mu(A)=\frac{d}{d t^{+}}\left\|e^{t A}\right\|_{t=0} .
$$

Proof. Eqn (21) follows from (20) and (19) with $t=0$.

We shall need the following lemma.

\section{Lemma 3.4.}

(i) The function $A \mapsto \mu(A)$ is Lipschitz continuous; that is, for any matrices $A$ and $B$, we have

$$
|\mu(A)-\mu(B)| \leq\|A-B\| .
$$

(ii) For any $z \in \mathbb{C}$, we have

$$
\mu(A+z I)=\mu(A)+\Re z
$$


Proof. (i) By (17) and (16), we have

$$
\begin{aligned}
& \mu(A) \leq \mu(A-B)+\mu(B) \leq\|A-B\|+\mu(B), \\
& \mu(B) \leq \mu(B-A)+\mu(A) \leq\|A-B\|+\mu(A),
\end{aligned}
$$

and the statement follows.

(ii) We have

$$
\begin{aligned}
\|I+h(A+z I)\| & =|1+h z|\left\|I+\frac{h}{1+h z} A\right\| \\
& =|1+h z|\left\|I+\frac{h(1+h \Re z-i h \Im z)}{|1+h z|^{2}} A\right\| \\
& =|1+h z|\left\|I+\frac{h(1+h \Re z)}{|1+h z|^{2}} A-\frac{h^{2} i \Im z}{|1+h z|^{2}} A\right\| .
\end{aligned}
$$

Hence, by (i)

$$
\begin{gathered}
\lim _{h \rightarrow 0^{+}} \frac{\|I+h(A+z I)\|-1}{h}=\lim _{h \rightarrow 0^{+}} \frac{|1+h z|\left\|I+\frac{h(1+h \Re z)}{|1+h z|^{2}} A\right\|-1}{h} \\
=\lim _{h \rightarrow 0^{+}} \frac{(1+h \Re z)}{|1+h z|} \frac{\left\|I+\frac{h(1+h \Re z)}{|1+h z|^{2}} A\right\|-1+1-\frac{1}{|1+h z|}}{\frac{h(1+h \Re z)}{|1+h z|^{2}}} .
\end{gathered}
$$

Next,

$$
1-\frac{1}{|1+h z|}=\frac{\sqrt{(1+h \Re z)^{2}+h^{2} \Im z^{2}}-1}{|1+h z|}=h \Re z+O\left(h^{2}\right)
$$

and the thesis follows as $0<\frac{1+h \Re z}{|1+h z|} \rightarrow 1$ as $h \rightarrow 0$.

Theorem 3.5. The logarithmic norm satisfies $\mu(A) \leq 0$ if and only if the semigroup $\left(e^{t A}\right)_{t \geq 0}$ is quasi-contractive. Precisely, $\left\|e^{t A}\right\| \leq e^{\mu(A) t}$ and $\mu(A)=\min \left\{\omega ;\left\|e^{t A}\right\| \leq e^{\omega t}\right\}$.

Proof. By Lemma 3.4 (ii), we have $\mu(A-\mu(A) I)=0$ and thus, by Theorem 3.2, $1 \geq\left\|e^{t(A-\mu(A) I)}\right\|=e^{-\mu(A) t}\left\|e^{t A}\right\|$, or $\left\|e^{t A}\right\| \leq e^{\mu(A) t}$. Conversely, if $\left\|e^{t A}\right\| \leq e^{\omega t}$, then $\left\|e^{t(A-\omega I)}\right\| \leq 1$ and, again by Lemma 3.4 (ii), $0 \geq \mu(A-\omega I)=\mu(A)-\omega$; that is, $\mu(A) \leq \omega$.

One of the drawbacks of the logarithmic norm is that it strongly depends on the underlying norm, see e.g. [2, Section 1 of Chapter 2], and thus it is not directly related to the dynamical properties of $\left(e^{t A}\right)_{t \geq 0}$. More precisely, if $\mu(A)<0$, then $\left(e^{t A}\right)_{t \geq 0}$ is an exponentially stable contractive semigroup. On the other hand, if $\left(e^{t A}\right)_{t \geq 0}$ is only exponentially stable; that is, for some $M \geq 1, \omega<0$

$$
\left\|e^{t A}\right\| \leq M e^{\omega t}
$$


for all $t \geq 0$, then nothing can be said about $\mu(A)$ for a given norm $\|\cdot\|$. We observe that in finite dimensional spaces the stability (24) is determined by

$$
s(A):=\max \{\Re \lambda ; \lambda \in \sigma(A)\},
$$

where $\sigma(A)$ is the spectrum of $A$, which is independent of the norm. We have, however,

Theorem 3.6. For any $\epsilon>0$ there is a norm $\|\cdot\|_{\epsilon}$ (equivalent to $\|\cdot\|$ ) such that the logarithmic norm $\mu_{\epsilon}$ defined by $\|\cdot\|_{\epsilon}$ satisfies

$$
s(A) \leq \mu_{\epsilon}(A) \leq s(A)+\epsilon .
$$

Further, if $s(A)$ is a semi-simple eigenvalue of $A$, then we can take $\epsilon=0$.

Proof. The first inequality follows from Theorem 3.5. Indeed, if $\lambda \in \sigma(A)$ satisfies $\Re \lambda=s(A)$, then for the normalized eigenvector $\boldsymbol{e}$ belonging to $\lambda$ we have

$$
\left\|e^{t A} \boldsymbol{e}\right\|=e^{t \Re \lambda}=e^{t s(A)}
$$

so that

$$
e^{t \mu(A)} \geq\left\|e^{t A}\right\| \geq\left\|e^{t A} \boldsymbol{e}\right\|=e^{t s(A)} .
$$

From general theory of linear systems, see e.g. [2, Chapter III, Eqn. (11)], for any $\epsilon>0$ there is $M_{\epsilon}$ such that

$$
\left\|e^{t A}\right\| \leq M_{\epsilon} e^{t(s(A)+\epsilon)},
$$

thus

$$
\left\|e^{t A_{\epsilon}}\right\|:=\left\|e^{t(A-(s(A)+\epsilon) I}\right\| \leq M_{\epsilon} .
$$

Defining an equivalent norm, see [4, Lemma II.3.10], by

$$
\|\boldsymbol{x}\|_{\epsilon}:=\sup _{s \in \mathbb{R}_{+}}\left\|e^{s A_{\epsilon}} \boldsymbol{x}\right\|,
$$

we see that

$$
\left\|e^{t A_{\epsilon}} \boldsymbol{x}\right\|_{\epsilon}=\sup _{s \in \mathbb{R}_{+}}\left\|e^{s A_{\epsilon}} e^{t A_{\epsilon}} \boldsymbol{x}\right\|=\sup _{s \in \mathbb{R}_{+}}\left\|e^{(s+t) A_{\epsilon}} \boldsymbol{x}\right\| \leq\|\boldsymbol{x}\|_{\epsilon},
$$

hence $\left(e^{t A_{\epsilon}}\right)_{t>0}$ is contractive and, by Theorem $3.2, \mu_{\epsilon}\left(A_{\epsilon}\right) \leq 0$. Thus, by Lemma 3.4 (ii),

$$
\mu_{\epsilon}(A) \leq s(A)+\epsilon .
$$

If all $\lambda \in \sigma(A)$ with $\Re \lambda=s(A)$ are (semi)-simple, then (27) is valid with $\epsilon=0$ and the second statement of the theorem follows.

Remark 3.7. Theorem 3.6 goes back possibly to $[9,10]$ but the proof above seems to be more elementary. On the other hand, the construction of [10] gives an inner product norm $\sqrt{\left(\boldsymbol{x}, P_{\epsilon} \boldsymbol{x}\right)}$, where $P_{\epsilon}$ is the hermitian solution of the Lyapunov equation

$$
P_{\epsilon} A+A^{*} P_{\epsilon}=-I \text {. }
$$




\section{Regularly perturbed systems}

As mentioned in Introduction, we consider a family of Cauchy problems in $D \times\left[0, \epsilon_{0}\right)$, where $D \in \mathbb{R}^{n}$ is a open domain and $\epsilon_{0}>0$,

$$
\begin{aligned}
\boldsymbol{x}_{\epsilon}^{\prime}(t) & =\boldsymbol{f}\left(\boldsymbol{x}_{\epsilon}(t), \epsilon\right), \quad t>0, \\
\boldsymbol{x}_{\epsilon}(0) & =\stackrel{\circ}{\boldsymbol{x}}_{\epsilon},
\end{aligned}
$$

with $\stackrel{x}{\epsilon}_{\epsilon} \in D$ for $\epsilon \in\left[0, \epsilon_{0}\right)$. We assume that $\boldsymbol{f}(\cdot, \epsilon) \in C_{b}^{2}(D)$ with respect to $\boldsymbol{x}$ uniformly in $\epsilon \in\left[0, \epsilon_{0}\right)$ and $\boldsymbol{f}(\boldsymbol{x}, \cdot) \in C_{b}^{1}\left(\left[0, \epsilon_{0}\right)\right)$ uniformly in $\boldsymbol{x} \in D$. We denote the semiflow generated by $(28)$ by $\boldsymbol{x}_{\epsilon}(t, \stackrel{\boldsymbol{x}}{)}$ and use the simplified notation $\boldsymbol{x}(t, \stackrel{\circ}{\boldsymbol{x}}):=\boldsymbol{x}_{0}(t, \stackrel{\circ}{\boldsymbol{x}})$.

Theorem 4.1. Assume that $\boldsymbol{x}^{*} \in D$ is a stationary solution of the limit equation

$$
\boldsymbol{x}^{\prime}(t)=\boldsymbol{f}(\boldsymbol{x}, 0), \quad t>0,
$$

and the spectral bound of the Jacobian $\mathcal{J}$ of $\boldsymbol{f}\left(\boldsymbol{x}^{*}, 0\right), s\left(\mathcal{J} \boldsymbol{f}\left(\boldsymbol{x}^{*}, 0\right)\right)$, satisfies $s\left(\mathcal{J} \boldsymbol{f}\left(\boldsymbol{x}^{*}, 0\right)\right)<0$. Further, let $\lim _{\epsilon \rightarrow 0^{+}} \stackrel{\circ}{\epsilon}_{\epsilon}=\stackrel{\boldsymbol{x}}{\boldsymbol{x}} \in D$ with $\stackrel{\circ}{\boldsymbol{x}}$ belonging to the domain of attraction of $\boldsymbol{x}^{*}$. Then

$$
\lim _{\epsilon \rightarrow 0^{+}} \boldsymbol{x}_{\epsilon}\left(t, \stackrel{\circ}{\boldsymbol{x}}_{\epsilon}\right)=\boldsymbol{x}(t, \stackrel{\stackrel{x}{x})}{ }
$$

uniformly on $[0, \infty)$.

Proof. To fix notation, let $\mathbb{R}^{n}$ be normed with the $l^{1}$ norm $\|\cdot\|_{1}$ and let $\|\cdot\|$ be a fixed equivalent norm on $\mathbb{R}^{n}$ such that the logarithmic norm of $\mathcal{J} \boldsymbol{f}\left(\boldsymbol{x}^{*}, 0\right)$ with respect to $\|\cdot\|$ satisfies $\mu\left(\mathcal{J} \boldsymbol{f}\left(\boldsymbol{x}^{*}, 0\right)\right)<0$, see Theorem 3.6. Let us specify

$$
c\|x\|_{1} \leq\|x\| \leq C\|x\|_{1}
$$

for some $c, C>0$. .

Using (22) and the regularity of $\boldsymbol{f}$, we find that there are $\delta_{1}>0,0<$ $\epsilon_{1}<\epsilon_{0}$, such that $B\left(\boldsymbol{x}^{*}, \delta_{1}\right) \subset D$ and

$$
\mu(\mathcal{J} \boldsymbol{f}(\boldsymbol{x}, \epsilon)) \leq \mu^{*}<0
$$

for $(\boldsymbol{x}, \epsilon) \in B\left(\boldsymbol{x}^{*}, \delta_{1}\right) \times\left[0, \epsilon_{1}\right)$.

Before we proceed, we need to combine the estimates for the logarithmic norm with some estimates of the second order remainder of the expansion of $\boldsymbol{f}$. For arbitrary $\boldsymbol{u}, \boldsymbol{v} \in D$, we have

$$
\boldsymbol{f}(\boldsymbol{u}, \epsilon)=\boldsymbol{f}(\boldsymbol{v}, \epsilon)+\mathcal{J} \boldsymbol{f}(\boldsymbol{v}, \epsilon)(\boldsymbol{u}-\boldsymbol{v})+R(\boldsymbol{u}-\boldsymbol{v}, \epsilon)(\boldsymbol{v}-\boldsymbol{u}),
$$

where

$$
R(\boldsymbol{u}-\boldsymbol{v}, \epsilon)=O(\|\boldsymbol{u}-\boldsymbol{v}\|), \quad\|\boldsymbol{u}-\boldsymbol{v}\| \rightarrow 0,
$$

uniformly in $\epsilon$. Indeed, suppressing in the notation $\epsilon$ as not relevant for the discussion, we proceed as in the classical proof of the multivariate Taylor 
expansion and consider the function $\phi=\left(\phi_{1}, \ldots, \phi_{n}\right)$, where the scalar functions $\phi_{i}, 1 \leq i \leq n$, are defined as

$$
\phi_{i}(s)=f_{i}(\boldsymbol{v}+s(\boldsymbol{u}-\boldsymbol{v})), \quad s \in[0,1],
$$

so that $\phi(0)=\boldsymbol{f}(\boldsymbol{v})$ and $\phi(1)=\boldsymbol{f}(\boldsymbol{u})$. Using the Taylor expansion with the integral remainder,

$$
\phi_{i}(1)=\phi_{i}(0)+\phi_{i}^{\prime}(0)+\int_{0}^{1}(1-\sigma) \phi_{i}^{\prime \prime}(\sigma) d \sigma,
$$

upon using

$$
\begin{aligned}
\phi_{i}^{\prime}(s) & =\sum_{j=1}^{n} \partial_{x_{j}} f_{i}(\boldsymbol{v}+s(\boldsymbol{u}-\boldsymbol{v}))\left(u_{j}-v_{j}\right), \\
\phi_{i}^{\prime \prime}(s) & =\sum_{j, k=1}^{n} \partial_{x_{j} x_{k}}^{2} f_{i}(\boldsymbol{v}+s(\boldsymbol{u}-\boldsymbol{v}))\left(u_{j}-v_{j}\right)\left(u_{k}-v_{k}\right),
\end{aligned}
$$

in the original notation we get

$$
f_{i}(\boldsymbol{u})=f_{i}(\boldsymbol{v})+D f_{i}(\boldsymbol{v})(\boldsymbol{u}-\boldsymbol{v})+\left\langle H_{i}(\boldsymbol{u}, \boldsymbol{v})(\boldsymbol{u}-\boldsymbol{v}),(\boldsymbol{u}-\boldsymbol{v})\right\rangle,
$$

where

$$
H_{i}(\boldsymbol{u}, \boldsymbol{v})=\int_{0}^{1}(1-\sigma)\left(\mathcal{H} f_{i}\right)(\boldsymbol{v}+\sigma(\boldsymbol{u}-\boldsymbol{v})) d \sigma
$$

and $\mathcal{H} f_{i}$ is the Hessian of $f_{i}$. Let us now return to the (uniform) dependence on $\epsilon$ and write down the complete estimate in vector notation. To do this, we introduce $\mathfrak{H}=\left\{\mathfrak{H}_{i k}\right\}_{1 \leq i, j \leq n}$ by

$$
\begin{aligned}
\mathfrak{H}_{i k}(\boldsymbol{u}, \boldsymbol{v}, \epsilon) & =\left(H_{i}(\boldsymbol{u}, \boldsymbol{v}, \epsilon)(\boldsymbol{u}-\boldsymbol{v})\right)_{k} \\
& =\int_{0}^{1}(1-\sigma) \sum_{j}^{n} \partial_{x_{j} x_{k}}^{2} f_{i}(\boldsymbol{v}+\sigma(\boldsymbol{u}-\boldsymbol{v}), \epsilon)\left(u_{j}-v_{j}\right) d \sigma
\end{aligned}
$$

so that

$$
\boldsymbol{f}(\boldsymbol{u}, \epsilon)=\boldsymbol{f}(\boldsymbol{v}, \epsilon)+\mathcal{J} \boldsymbol{f}(\boldsymbol{v}, \epsilon)(\boldsymbol{u}-\boldsymbol{v})+\mathfrak{H}(\boldsymbol{u}, \boldsymbol{v}, \epsilon)(\boldsymbol{u}-\boldsymbol{v}),
$$

where

$$
\|\mathfrak{H}(\boldsymbol{u}, \boldsymbol{v}, \epsilon)\| \leq M\|\boldsymbol{u}-\boldsymbol{v}\|
$$

here $M=\frac{C}{c^{2}} \frac{L n}{2}$,

$$
L=\sup \left\{\left|\partial_{x_{j} x_{k}}^{2} f_{i}(\boldsymbol{x}, \epsilon)\right| ; \boldsymbol{x} \in D, \epsilon \in\left[0, \epsilon_{0}\right), i, j, k=1, \ldots, n\right\}
$$

and $C, c$ were defined in (31). We see that there is $\delta<\delta_{1}$ such that

$$
\mu^{*}+M \delta \leq \bar{\mu}<0
$$


for some $\bar{\mu}$. By assumption, there is $T$ such that for all $t \geq T$ we have $\boldsymbol{x}\left(t, \stackrel{\boldsymbol{x}}{)} \in B\left(\boldsymbol{x}^{*}, \frac{\delta}{4}\right)\right.$. Then, by the regular perturbation theorem, Theorem 1.1, for any $0<\eta<\frac{\delta}{4}$ there is $\epsilon_{\eta}>0$ such that for all $\epsilon \in\left[0, \epsilon_{\eta}\right)$ and $t \in[0, T]$ we have

$$
\left\|\boldsymbol{x}_{\epsilon}\left(t, \stackrel{\circ}{\boldsymbol{x}}_{\epsilon}\right)-\boldsymbol{x}(t, \stackrel{\circ}{\boldsymbol{x}})\right\| \leq \eta .
$$

Thus, $\boldsymbol{x}_{\epsilon}\left(T, \stackrel{\circ}{\boldsymbol{x}}_{\epsilon}\right) \in B\left(\boldsymbol{x}^{*}, \frac{\delta}{2}\right)$ for $\epsilon<\epsilon_{\eta}$.

Next, following the ideas of $[1,7]$, we introduce a $C_{0}^{\infty}\left(\mathbb{R}^{n}\right)$ cut-off function $0 \leq \psi \leq 1$ that satisfies

$$
\psi(\boldsymbol{x})= \begin{cases}1 & \text { for } \quad\left\|\boldsymbol{x}-\boldsymbol{x}^{*}\right\| \leq \delta \\ 0 & \text { for } \quad\left\|\boldsymbol{x}-\boldsymbol{x}^{*}\right\| \geq \delta\end{cases}
$$

Such a function exists by [16, Lemma 1 , Chapter $2, \S 5]$ and the fact that $\|\cdot\|$ is equivalent to the Euclidean norm used in the proof in op. cit. so that there is an Euclidean neighbourhood separating $B\left(\boldsymbol{x}^{*}, \frac{\delta}{2}\right)$ and the exterior of $B\left(\boldsymbol{x}^{*}, \delta\right)$. Shortening notation to $\boldsymbol{x}(t):=\boldsymbol{x}\left(t, \stackrel{\boldsymbol{x}}{)}\right.$ and $\boldsymbol{x}_{\epsilon}(t):=\boldsymbol{x}_{\epsilon}\left(t, \stackrel{\circ}{\boldsymbol{x}}_{\epsilon}\right)$, we consider the following modification of (28),

$$
\begin{aligned}
& \boldsymbol{y}_{\epsilon}^{\prime}=\widetilde{\boldsymbol{f}}\left(t, \boldsymbol{y}_{\epsilon}, \epsilon\right), \quad t>T, \\
& \boldsymbol{y}_{\epsilon}(T)=\boldsymbol{x}_{\epsilon}(T),
\end{aligned}
$$

where

$$
\begin{aligned}
\widetilde{\boldsymbol{f}}\left(t, \boldsymbol{y}_{\epsilon}, \epsilon\right):= & \boldsymbol{f}(\boldsymbol{x}(t), \epsilon)+\mathcal{J} \boldsymbol{f}(x, \epsilon)\left(\boldsymbol{y}_{\epsilon}-\boldsymbol{x}(t)\right) \\
& +\psi\left(\boldsymbol{y}_{\epsilon}-\boldsymbol{x}(t)\right) \mathfrak{H}\left(\boldsymbol{y}_{\epsilon}, \boldsymbol{x}(t), \epsilon\right)\left(\boldsymbol{y}_{\epsilon}-\boldsymbol{x}(t)\right) .
\end{aligned}
$$

We observe that $\boldsymbol{y}_{\epsilon}(t)=\boldsymbol{x}_{\epsilon}(t)$ as long as $\left\|\boldsymbol{y}_{\epsilon}(\tau)-\boldsymbol{x}(\tau)\right\| \leq \frac{\delta}{2}$ for $T \leq \tau \leq t$.

Now, as in [5, Section I.10], we derive a differential inequality for the norm of the error $\boldsymbol{e}_{\epsilon}(t)=\boldsymbol{y}_{\epsilon}(t)-\boldsymbol{x}(t), t \geq T$. For a fixed $t \geq T$, we have

$$
\begin{aligned}
\left\|\boldsymbol{e}_{\epsilon}(t+h)\right\|= & \left\|\boldsymbol{y}_{\epsilon}(t+h)-\boldsymbol{x}(t+h)\right\| \leq \| \boldsymbol{e}_{\epsilon}(t)+h\left(\boldsymbol{y}_{\epsilon}^{\prime}(t)-\boldsymbol{x}^{\prime}(t)+O\left(h^{2}\right) \|\right. \\
\leq & \left\|\boldsymbol{e}_{\epsilon}(t)+h\left(\widetilde{\boldsymbol{f}}\left(t, \boldsymbol{y}_{\epsilon}(t), \epsilon\right)-\boldsymbol{f}(\boldsymbol{x}(t), 0)\right)\right\|+O\left(h^{2}\right) \\
\leq & \left\|\boldsymbol{e}_{\epsilon}(t)+h\left(\widetilde{\boldsymbol{f}}\left(t, \boldsymbol{y}_{\epsilon}(t), \epsilon\right)-\boldsymbol{f}(\boldsymbol{x}(t), \epsilon)\right)\right\| \\
& +h\|\boldsymbol{f}(\boldsymbol{x}(t), \epsilon)-\boldsymbol{f}(\boldsymbol{x}(t), 0)\|+O\left(h^{2}\right) \\
\leq & \left\|I+h\left(\mathcal{J} \boldsymbol{f}(\boldsymbol{x}, \epsilon)+\psi\left(\boldsymbol{y}_{\epsilon}(t)-\boldsymbol{x}(t)\right) \mathfrak{H}\left(\boldsymbol{y}_{\epsilon}(t), \boldsymbol{x}(t), \epsilon\right)\right)\right\|\left\|\boldsymbol{e}_{\epsilon}(t)\right\| \\
& +\epsilon h K+O\left(h^{2}\right),
\end{aligned}
$$

where $K=\sup \left\{\left\|\partial_{\epsilon} \boldsymbol{f}(\boldsymbol{x}, \epsilon)\right\| ; \boldsymbol{x} \in B\left(\boldsymbol{x}^{*}, \frac{\delta}{4}\right), \epsilon \in\left[0, \epsilon_{0}\right)\right\}$. Now, subtracting $\left\|\boldsymbol{e}_{\epsilon}(t)\right\|$ from both sides, dividing by $h$ and passing to the limit as $h \rightarrow 0^{+}$, we obtain, by Corollary 2.7, for any fixed $t \geq T$,

$$
\begin{aligned}
\left\|\boldsymbol{e}_{\epsilon}(t)\right\|_{+}^{\prime} \leq & \mu\left(\mathcal{J} \boldsymbol{f}(\boldsymbol{x}, \epsilon)+\psi\left(\boldsymbol{y}_{\epsilon}(t)-\boldsymbol{x}(t)\right) \mathfrak{H}\left(\boldsymbol{y}_{\epsilon}(t), \boldsymbol{x}(t), \epsilon\right)\right)\left\|\boldsymbol{e}_{\epsilon}(t)\right\| \\
& +\epsilon K
\end{aligned}
$$


Next, using the definition of $\psi$, we estimate:

(i) For $\left\|\boldsymbol{y}_{\epsilon}(t)-\boldsymbol{x}(t)\right\| \leq \frac{\delta}{2}$, by (32),

$$
\begin{gathered}
\mu\left(\mathcal{J} \boldsymbol{f}(\boldsymbol{x}, \epsilon)+\psi\left(\boldsymbol{y}_{\epsilon}(t)-\boldsymbol{x}(t)\right) \mathfrak{H}\left(\boldsymbol{y}_{\epsilon}(t), \boldsymbol{x}(t), \epsilon\right)\right)=\mu\left(\mathcal{J} \boldsymbol{f}\left(\boldsymbol{y}_{\epsilon}(t), \epsilon\right)\right) \leq \mu^{*}<0, \\
\text { as }\left\|\boldsymbol{y}_{\epsilon}(t)-\boldsymbol{x}^{*}\right\| \leq\left\|\boldsymbol{y}_{\epsilon}(t)-\boldsymbol{x}(t)\right\|+\left\|\boldsymbol{x}(t)-\boldsymbol{x}^{*}\right\| \leq \delta<\delta_{1} . \\
\left(\text { ii) } \text { For } \frac{\delta}{2}<\left\|\boldsymbol{y}_{\epsilon}(t)-\boldsymbol{x}(t)\right\| \leq \delta, \text { using }(22) \text { and }(37),\right. \\
\mu\left(\mathcal{J} \boldsymbol{f}(\boldsymbol{x}, \epsilon)+\psi\left(\boldsymbol{y}_{\epsilon}(t)-\boldsymbol{x}(t)\right) \mathfrak{H}\left(\boldsymbol{y}_{\epsilon}(t), \boldsymbol{x}(t), \epsilon\right)\right) \\
\leq \mu(\mathcal{J} \boldsymbol{f}(\boldsymbol{x}, \epsilon))+\left\|\mathfrak{H}\left(\boldsymbol{y}_{\epsilon}(t), \boldsymbol{x}(t), \epsilon\right)\right\| \leq \bar{\mu}<0,
\end{gathered}
$$

as $0 \leq \psi \leq 1$.

(iii) For $\left\|\boldsymbol{y}_{\epsilon}(t)-\boldsymbol{x}(t)\right\|>\delta$, again by (32),

$$
\begin{aligned}
\mu(\mathcal{J} \boldsymbol{f}(\boldsymbol{x}, \epsilon) & \left.+\psi\left(\boldsymbol{y}_{\epsilon}(t)-\boldsymbol{x}(t)\right) \mathfrak{H}\left(\boldsymbol{y}_{\epsilon}(t), \boldsymbol{x}(t), \epsilon\right)\right)=\mu(\mathcal{J} \boldsymbol{f}(\boldsymbol{x}(t), \epsilon)) \leq \mu^{*} \\
& \text { as } \boldsymbol{x}(t) \in B\left(\boldsymbol{x}^{*}, \frac{\delta}{4}\right) \text { for } t \geq T .
\end{aligned}
$$

Since $0>\bar{\mu}>\mu^{*}$, we obtain the following differential inequality for the norm of the error

$$
\begin{aligned}
\left\|\boldsymbol{e}_{\epsilon}(t)\right\|_{+}^{\prime} & \leq \bar{\mu}\left\|\boldsymbol{e}_{\epsilon}(t)\right\|+\epsilon K, \quad t \geq T \\
\left\|\boldsymbol{e}_{\epsilon}(T)\right\| & =\left\|\boldsymbol{x}_{\epsilon}(T)-\boldsymbol{x}(T)\right\| .
\end{aligned}
$$

Since scalar equations automatically are of type $K$, from Remark 2.6 we obtain

$$
\begin{aligned}
\left\|\boldsymbol{e}_{\epsilon}(t)\right\| & \leq e^{\bar{\mu}(t-T)}\left\|\boldsymbol{x}_{\epsilon}(T)-\boldsymbol{x}(T)\right\|+\epsilon \frac{K}{-\bar{\mu}}\left(1-e^{\bar{\mu}(t-T)}\right) \\
& \leq\left\|\boldsymbol{x}_{\epsilon}(T)-\boldsymbol{x}(T)\right\|+\epsilon \frac{K}{-\bar{\mu}}, \quad t \geq T .
\end{aligned}
$$

Thus, defining $\epsilon_{3}:=\frac{-\bar{\mu} \delta}{2 K},(38)$ implies that $\left\|\boldsymbol{y}_{\epsilon}(t)-\boldsymbol{x}(t)\right\| \leq \frac{\delta}{2}$ for any $\epsilon \in\left[0, \epsilon_{3}\right)$ and hence, for such $\epsilon, \boldsymbol{y}_{\epsilon}(t)=\boldsymbol{x}_{\epsilon}(t)$. Therefore, (42) can be written as

$$
\left\|\boldsymbol{x}_{\epsilon}(t)-\boldsymbol{x}(t)\right\| \leq\left\|\boldsymbol{x}_{\epsilon}(T)-\boldsymbol{x}(T)\right\|+\epsilon \frac{K}{-\bar{\mu}}, \quad t \geq T .
$$

Combining (38) and (43), we obtain that for any (sufficiently small) $\eta$ there is $\epsilon_{4}<\min \left\{\epsilon_{3}, \epsilon_{\eta}, \eta \frac{-\bar{\mu}}{K}\right\}$ such that for all $0<\epsilon<\epsilon_{4}$ and $t \in[0, \infty)$

$$
\left\|\boldsymbol{x}_{\epsilon}\left(t, \stackrel{\circ}{\boldsymbol{x}}_{\epsilon}\right)-\boldsymbol{x}(t, \stackrel{\circ}{\boldsymbol{x}})\right\| \leq 3 \eta,
$$

proving the uniform convergence of $\boldsymbol{x}_{\epsilon}(t)$ to $\boldsymbol{x}(t)$ on $[0, \infty)$.

Example 4.2. To illustrate the result, let us consider the Ross model of malaria, as derived in [11],

$$
\begin{aligned}
& I_{h}^{\prime}=b_{1} a I_{v} \frac{H-I_{h}}{H}-\left(\gamma_{H}+\mu_{H}\right) I_{h}, \\
& I_{v}^{\prime}=b_{2} a\left(V-I_{v}\right) \frac{I_{h}}{H}-\left(\gamma_{V}+\mu_{V}\right) I_{v},
\end{aligned}
$$


where $I_{h}$ and $I_{v}$ are, respectively, the numbers of infected humans and mosquitoes, $H$ and $V$ are, respectively, the total populations of humans and mosquitoes, $b_{1}$ is the probability of a human becoming infectious after an infectious bite, $a$ is the rate of bites of infectious mosquitoes, $\gamma_{H}$ is the human rate of recovery, $\mu_{H}$ is the human death rate, $b_{2}$ is the probability that a mosquito becomes infectious after an infectious bite, while $\gamma_{V}$ and $\mu_{V}$ are, respectively, the recovery and death rates of infected mosquitoes. Since it is assumed that the human and mosquito populations are constant, we normalize them by setting $x=I_{h} / H$ and $y=I_{v} / V$ so that (44) becomes

$$
\begin{aligned}
x^{\prime} & =m b_{1} a y(1-x)-\left(\gamma_{H}+\mu_{H}\right) x, \\
y^{\prime} & =b_{2} a(1-y) x-\left(\gamma_{V}+\mu_{V}\right) y,
\end{aligned}
$$

where $m=V / H$. Furthermore, it is observed that human mortality is negligible on the time scale of the recovery rate. Also, in epidemiological literature it is assumed that mosquitoes remain infected practically for the whole life after becoming infectious. Thus, using $\mu_{H}=\gamma_{V} \approx 0$, (45) can be written as

$$
\begin{aligned}
& x^{\prime}=m b_{1} a y(1-x)-\gamma_{H} x, \\
& y^{\prime}=b_{2} a(1-y) x-\mu_{V} y .
\end{aligned}
$$

Can we say that that the dynamics described by (45) does not change, when we simplify it to (46)? To illustrate the application of Theorem 4.1, let us focus on the disease free equilibrium $(0,0)$. The Jacobian at this point is given by

$$
\mathcal{J}=\left(\begin{array}{cc}
-\gamma_{H} & m a b_{1} \\
a b_{2} & -\mu_{V}
\end{array}\right),
$$

with the characteristic equation $\lambda^{2}+\left(\gamma_{H}+\mu_{V}\right) \lambda-m a^{2} b_{1} b_{2}+\gamma_{H} \mu_{V}=0$. Since $\Delta=\left(\gamma_{H}-\mu_{V}\right)^{2}+m a^{2} b_{1} b_{2}>0$, if

$$
\frac{m a^{2} b_{1} b_{2}}{\gamma_{H} \mu_{V}}<1,
$$

then $s(\mathcal{J})<0$ and $(0,0)$ is asymptotically stable (in fact, globally asymptotically stable in $[0,1] \times[0,1]$, see $[11$, Theorem 2.3.1]). Other assumptions being satisfied, Theorem 4.1 (subject to a small modification to allow for the fact that $\mu_{H}$ and $\gamma_{V}$ are not necessarily functions of a single parameter $\epsilon$ ) asserts that the solutions to (45) originating from $(\stackrel{\circ}{x}, \stackrel{\circ}{y}) \in[0,1] \times[0,1]$ converge to the solution to (46) with the same initial condition, uniformly on $[0, \infty)$ as $\gamma_{H}, \mu_{V} \rightarrow 0$.

We mention that while in the epidemiological context this result is not unexpected as we dropped negative terms on the right hand side of (45) so that the solutions to (46) decay to zero faster than those of (46) mathematically, however, the result does not depend on the sign of $\mu_{H}$ and $\gamma_{V}$. 


\section{References}

[1] Carr, J., Applications of Centre Manifold Theory, Applied Mathematical Sciences 35, Springer-Verlag, New York-Berlin, 1981.

[2] Coppel, W. A., Stability and Asymptotic Behavior of Differential Equations, D. C. Heath and Co., Boston, Mass., 1965.

[3] Dahlquist, G., Stability and error bounds in the numerical integration of ordinary differential equations, Kungl. Tekn. Högsk. Handl. Stockholm. No. 130 (1959), 87pp.

[4] Engel, K.-J., Nagel, R., One-parameter Semigroups for Linear Evolution Equations, Graduate Texts in Mathematics 194, Springer-Verlag, New York, 2000.

[5] Hairer, E., Nørsett, S. P., Wanner, G., Solving Ordinary Differential Equations. I. Nonstiff Problems, Springer Series in Computational Mathematics 8, Springer-Verlag, Berlin, second edition, 1993.

[6] Lozinskiǔ, S. M., Error estimate for numerical integration of ordinary differential equations. I, Izv. Vysš. Učebn. Zaved. Matematika 5 (6) (1958), 52-90 (Russian); 5 (12) (1959), 222 (Erratum).

[7] Marciniak-Czochra, A., Mikelić, A., Stiehl, T., Renormalization group second-order approximation for singularly perturbed nonlinear ordinary differential equations, Math. Methods Appl. Sci. 41 (14) (2018), 5691-5710.

[8] Ortega, J. M., Numerical Analysis, Classics in Applied Mathematics 3, Society for Industrial and Applied Mathematics (SIAM), Philadelphia, PA, second edition, 1990.

[9] Pao, C. V., A further remark on the logarithmic derivatives of a square matrix, Linear Algebra and Appl. 7 (1973), 275-278.

[10] Pao, C. V., Logarithmic derivates of a square matrix, Linear Algebra and Appl. 6 (1973), 159-164.

[11] Sallet, G., Mathematical Epidemiology, Lecture Notes, 2018. http://www.iecl.univ-lorraine.fr/Gauthier.Sallet/Lecture-Notes-Pretoria-2018.pdf

[12] Smith, H. L., Waltman, P., The Theory of the Chemostat, Cambridge Studies in Mathematical Biology 13, Cambridge University Press, Cambridge, 1995.

[13] Söderlind, G., The logarithmic norm. History and modern theory, BIT 46 (3) (2006), 631-652.

[14] Ström, T., On logarithmic norms, SIAM J. Numer. Anal. 12 (5) (1975), 741-753.

[15] Uherka, D. J., Sergott, A. M., On the continuous dependence of the roots of a polynomial on its coefficients, Amer. Math. Monthly 84 (5) (1977), 368-370.

[16] Vladimirov, V. S., Equations of Mathematical Physics, Pure and Applied Mathematics 3, Marcel Dekker, Inc., New York, 1971.

[17] Walter, W., Ordinary Differential Equations, Graduate Texts in Mathematics 182, Springer-Verlag, New York, 1998.

Jacek Banasiak

Department of Mathematics and Applied Mathematics

University of Pretoria

South Africa

Institute of Mathematics

Łódź University of Technology

Poland

e-mail: jacek. banasiak@up.ac.za

Received July 31, 2019 